\title{
Structural, vibrational, and electrical study of compressed BiTeBr
}

\author{
J. A. Sans,,${ }^{1, *}$ F. J. Manjón, ${ }^{1}$ A. L. J. Pereira,,${ }^{1,2}$ R. Vilaplana, ${ }^{3}$ O. Gomis,${ }^{3}$ A. Segura, ${ }^{4}$ A. Muñoz, ${ }^{5}$ P. Rodríguez-Hernández, ${ }^{5}$ \\ C. Popescu, ${ }^{6}$ C. Drasar, ${ }^{7}$ and P. Ruleova ${ }^{7}$ \\ ${ }^{1}$ Instituto de Diseño para la Fabricación y Producción Automatizada, Universitat Politècnica de València, 46022 Valencia, Spain \\ ${ }^{2}$ Laboratório de Materiais Cerâmicos Avançados, Faculdade de Ciências Exatas e Tecnologia, Universidade Federal da Grande Dourados, \\ Dourados, Brazil \\ ${ }^{3}$ Centro de Tecnologías Físicas, Universitat Politècnica de València, 46022 Valencia, Spain \\ ${ }^{4}$ Instituto de Ciencia de Materiales de la Universidad de Valencia, Departamento de Física Aplicada, Universitat de València, \\ 46100 Burjassot, Valencia, Spain \\ ${ }^{5}$ Departamento de Física, Instituto Universitario de Materiales y Nanotecnología, Universidad de La Laguna, La Laguna, Tenerife, Spain \\ ${ }^{6}$ ALBA-CELLS, 08290 Cerdanyola, Barcelona, Spain \\ ${ }^{7}$ Faculty of Chemical Technology, University of Pardubice, Studentská 95, 53210-Pardubice, Czech Republic \\ (Received 3 August 2015; revised manuscript received 31 October 2015; published 15 January 2016)
}

\begin{abstract}
Compresed $\mathrm{BiTeBr}$ has been studied from a joint experimental and theoretical perspective. Room-temperature $\mathrm{x}$-ray diffraction, Raman scattering, and transport measurements at high pressures have been performed in this layered semiconductor and interpreted with the help of $a b$ initio calculations. A reversible first-order phase transition has been observed above 6-7 GPa, but changes in structural, vibrational, and electrical properties have also been noted near $2 \mathrm{GPa}$. Structural and vibrational changes are likely due to the hardening of interlayer forces rather than to a second-order isostructural phase transition while electrical changes are mainly attributed to changes in the electron mobility. The possibility of a pressure-induced electronic topological transition and of a pressure-induced quantum topological phase transition in $\mathrm{BiTeBr}$ and other bismuth tellurohalides, like BiTeI, is also discussed.
\end{abstract}

DOI: 10.1103/PhysRevB.93.024110

\section{INTRODUCTION}

Bismuth tellurohalides $\operatorname{BiTe} X(X=\mathrm{Cl}, \mathrm{Br}, \mathrm{I})$ have received increasing attention in recent years because they are large Rashba semiconductors with a strong relevance for spintronics [1-6]. Rashba-induced spin splitting is the consequence of a strong spin-orbit interaction and inversion asymmetry, a narrow band gap, and the same symmetry of the topmost valence band and lowermost conduction band [7-9]. The interest in these materials has also recently increased because the spin texture in the momentum space of Rashba-type spin systems is similar to that of topological insulators [10] so their strong spin-orbit interaction could also lead to topological surface or bulk states. In fact, $\mathrm{BiTeCl}$ has been discovered to be the first strong inversion-asymmetric topological insulator $[5,6]$. The search for topological features in materials including topological insulators and superconductors is a hot topic because of its relevance for fundamental physics and applications in quantum computation [11,12].

Pressure is playing an important role in unraveling the mysteries of materials with strong spin-orbit interactions. High-pressure studies in topological insulators and Rashba semiconductors, like bismuth tellurohalides, are contributing enormously to the understanding of the physics of these complex layered semiconductors. In particular, a pressureinduced topological quantum phase transition (TQPT) has been claimed to occur in BiTeI on the basis of infrared data and density functional theory (DFT) calculations [13-15] which was subsequently questioned [16] on the basis of more infrared data and improved GW band structure calculations [17].

\footnotetext{
*Corresponding author: juasant2@upvnet.upv.es
}

Furthermore, several pressure-induced phase transitions have been discovered in BiTeI, the properties of which have not been studied in detail [14-16,18]. Additionally, $\mathrm{BiTeCl}$ has been recently found to become superconducting at high pressures [19], but whether it is the first topological superconductor ever observed is still under discussion. These examples just show that high-pressure studies are important to understand the exotic properties of bismuth tellurohalides.

Bismuth tellurobromide (BiTeBr), which is usually an $n$-type semiconductor, is the least studied of the three $\mathrm{BiTe} X$ compounds. Unlike $\mathrm{BiTeCl}$ and $\mathrm{BiTeI}, \mathrm{BiTeBr}$ was long thought to be the only disordered centrosymmetric bismuth tellurohalide crystallizing in the $\mathrm{CdI}_{2}$-type structure with space group (SG) $P \overline{3} m 1$ (no. 164) [20,21]. However, recent calculations have shown that $\mathrm{BiTeBr}$ is more stable in the ordered configuration; i.e., in the noncentrosymmetric BiTeItype crystal structure [Fig. 1(c)] with SG $P 3 m 1$ (no. 156) [22], in good agreement with recent experiments [4,23]. The noncentrosymmetric structure of $\mathrm{BiTeBr}$ with $\mathrm{SG} P 3 m 1$ is composed of triple layers $\mathrm{Te}-\mathrm{Bi}-\mathrm{Br}$ stacked along the polar $c$ axis, where atoms occupy $1 \mathrm{a}(\mathrm{Bi}), 1 \mathrm{~b}(\mathrm{Br})$, and $1 \mathrm{c}(\mathrm{Te})$ Wyckoff sites. On the other hand, the centrosymmetric structure with SG $P \overline{3} m 1$ is also composed of triple layers where atoms occupy $1 a(\mathrm{Bi})$ and $2 d(\mathrm{Br} / \mathrm{Te})$ sites; i.e., there is a mixture of $\mathrm{Br}$ and Te atoms at the two Wyckoff sites $(1 b$ and $1 c)$ of SG $P 3 m 1$ leading to a $2 d$ Wyckoff site in SG $P \overline{3} m 1$.

The interest in $\mathrm{BiTeBr}$ is increasing in recent years. It has been found to be a good thermoelectric material exhibiting a similar thermoelectric efficiency at room temperature as $\mathrm{Bi}_{2} \mathrm{Te}_{3}$, the leading compound of highly efficient thermoelectric materials operating near room temperature [24,25]. Furthermore, recent calculations show that BiTeBr has very interesting properties that feature a larger Rashba spin splitting 

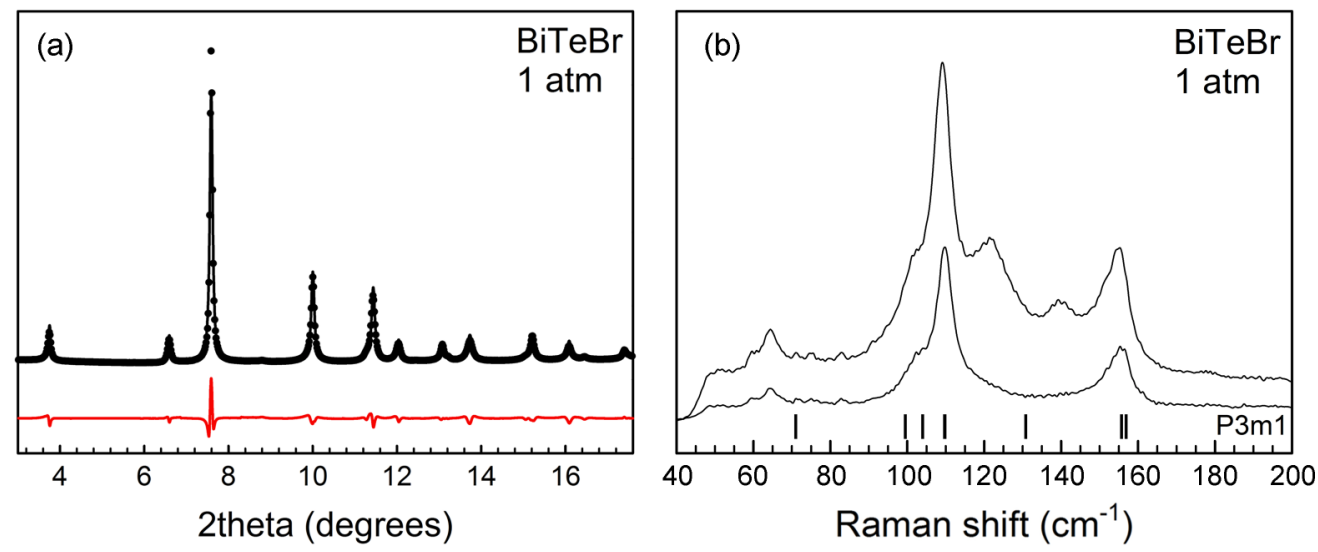

(c)

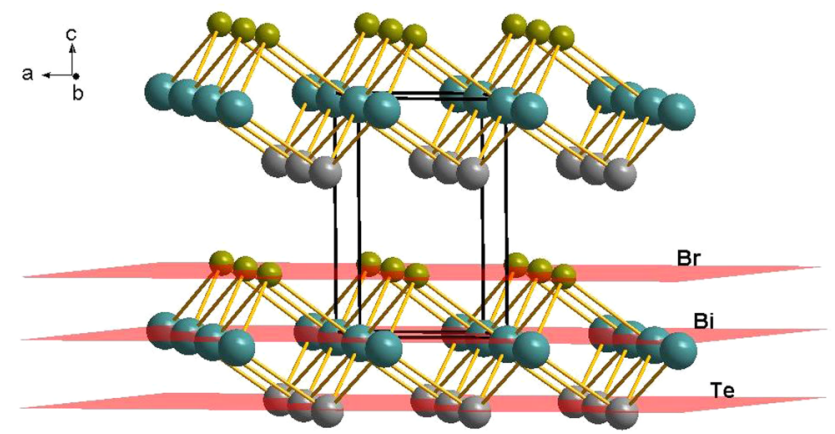

FIG. 1. (a) Synchrotron powder XRD pattern of BiTeBr at ambient pressure. Le Bail refinement and residuals are also plotted. (b) RS spectrum of BiTeBr at ambient pressure at two different excitation densities differing in a factor of 2.0: bottom, low-density excitation; top, high-density excitation. Theoretical frequencies of the first-order Raman-active modes are marked at the bottom for comparison. (c) Scheme of BiTeI-type BiTeBr.

and larger band gap than $\mathrm{BiTeCl}$ and a larger splitting off from the bulk conduction band with more isotropic energy dispersion within the band gap region than BiTeI [22]. Additionally, BiTeBr monolayers have been predicted to exhibit a stronger polar electric field than BiTeI, which is interesting for many applications [26].

In this work, we report the structural, vibrational, and electrical behavior of compressed BiTeBr (with BiTeI-type structure) by means of x-ray diffraction (XRD), Raman scattering (RS), and transport measurements at room temperature, which are compared and interpreted thanks to $a b$ initio calculations. Our results confirm the observation of a phase transition above 6-7 GPa and changes in the structural, vibrational, and electrical properties near $2 \mathrm{GPa}$, which are discussed in detail and in relation to a possible (i) isostructural phase transition (IPT), (ii) electronic topological transition (ETT) and (iii) topological quantum phase transition (TQPT).

\section{EXPERIMENTAL DETAILS}

Single crystals of BiTeBr were grown by a gas-phase transport reaction in a temperature gradient [27]. The starting stoichiometric mixtures of high-purity $\mathrm{BiBr}_{3}$ and $\mathrm{Bi}$ and Te elements (both 99.999\%) from Sigma-Aldrich were weighed in a glovebox into quartz glass ampoules and evacuated to pressure $1 \times 10^{-3}-2 \times 10^{-3} \mathrm{~Pa}$. The transport reaction was carried out in a horizontal furnace with gradient $480{ }^{\circ} \mathrm{C}-440^{\circ} \mathrm{C}$ per $20 \mathrm{~cm}$ for 9 days, leading to platelike single crystals with an area up to $100 \mathrm{~mm}^{2}$. Prior to the crystal growth process, a heating of the ampoules in reversed temperature gradient was employed to purify the growth side of the ampoules.

Angle-dispersive powder XRD experiments at room temperature at different pressures up to $20 \mathrm{GPa}$ were conducted in a membrane-type diamond anvil cell (DAC) at the BL04-MSPD beamline of ALBA synchrotron. The incident monochromatic beam with wavelength of $0.4246 \AA$ was focused to $20 \times 20 \mu \mathrm{m}^{2}$ using a pinhole of $50 \mu \mathrm{m}$ to cut the $\mathrm{x}$-ray beam tail [28]. Images covering a $2 \theta$ range up to $20^{\circ}$ were collected using a SX165 CCD located at $240 \mathrm{~mm}$ from the sample. One-dimensional diffraction profiles of intensity as a function of $2 \theta$ were obtained by integration of the observed intensities with the FIT2D software [29]. Because the strong preferred orientation of this laminar compound, revealed by the high-texture index obtained with GSAS software [30,31], affects the refined atomic parameters, these were considered unreliable. Therefore, the Le Bail method was also used to get structural parameters from powder XRD patterns. Interatomic distances from theoretical calculations were extracted with VESTA software [32]. Single crystals were crushed in a mortar with a pestle to obtain micron-sized powder for XRD measurements. The equation of state of copper [33], whose powder was mixed with the sample powder, was used for pressure calibration. A methanol-ethanol (4:1 ratio) mixture and silicone oil were used as pressure-transmitting media with quasihydrostatic conditions up to $10 \mathrm{GPa}[34,35]$. 
RS measurements were conducted with a Horiba Jobin Yvon LabRAM HR microspectrometer equipped with a thermoelectric-cooled multichannel CCD detector and with a spectral resolution below $2 \mathrm{~cm}^{-1}$. A HeNe laser (6328 $\AA$ line) with a power below $2 \mathrm{~mW}$ to avoid sample heating was used for excitation. Polarized and unpolarized RS measurements at room temperature and different pressures up to $7 \mathrm{GPa}$ were performed on a small flake of the single crystal inside the DAC. RS measurements were carried out in backscattering geometry for vertical [parallel or $(X X)$ ] and horizontal [perpendicular or $(X Y)]$ polarizations, where $X$ and $X$ (or $Y$ ) stand for the polarization directions of the incident and scattered light in the basal plane of the crystal, respectively. A methanolethanol (4:1 ratio) mixture was used as a pressure-transmitting medium. Pressure was determined by the ruby luminescence method [36].

Resistivity measurements at different pressures were carried out in a sample typically $200 \mu \mathrm{m}$ thick and $3 \times 3 \mathrm{~mm}^{2}$ in size mounted between two steel-belted Bridgman tungsten carbide (WC) anvils operated by a 150 ton oil press. Two experiments were performed with WC anvils having tips of 27 and $15 \mathrm{~mm}$, which reach a maximum pressure of 6 and $12 \mathrm{GPa}$, respectively [37]. Hall effect measurements were performed during the first experiment up to $3 \mathrm{GPa}$, but not in the second experiment up to $7 \mathrm{GPa}$. Hexagonal boron nitride (h-BN) was used as an insulating pressure-transmitting medium. Conductive epoxy contacts were deposited onto the sample corners in order to direct the current into the sample and measure the Ohmic signal. Linearity of the Ohmic voltages with the injected current was checked out at different pressures. Pressure was determined by the calibration of the load applied to the anvils against high-pressure resistivity transitions in the calibrants [38].

\section{Ab initio CALCULATIONS}

$A b$ initio total-energy calculations were performed for $\mathrm{BiTeBr}$ in the ordered structure with SG $P 3 m 1$ within the density functional theory (DFT) [39] using the plane-wave method and the pseudopotential theory with the Vienna $a b$ initio simulation package (VASP) [40] including scalar relativistic effects and spin-orbit interaction. The projector-augmented wave scheme [41] was used as implemented in this package, and the basis set of plane waves extended up to an energy cutoff of $320 \mathrm{eV}$ in order to achieve highly converged results and accurate description of the electronic properties. The exchange-correlation energy was described in the generalized gradient approximation with the PBEsol [42] prescription. In order to obtain very well-converged energies and forces, the integration over the Brillouin zone (BZ) was performed using dense meshes of special $k$ points. At selected volumes, the structures were fully relaxed to their optimized configuration through the calculation of the forces on atoms and the stress tensor. In the optimized configurations, the forces on the atoms are less than $0.002 \mathrm{eV} / \AA$ and the deviations of the stress tensor from a diagonal hydrostatic form are less than $1 \mathrm{kbar}(0.1 \mathrm{GPa})$. The application of DFT-based total-energy calculations to the study of semiconductor properties under high pressure has been reviewed, and it has been demonstrated that the phase stability and electronic and dynamical properties of
TABLE I. Theoretical fractional coordinates corresponding to the hexagonal phase $P 3 m 1$ of $\mathrm{BiTeBr}$ at room pressure. Theoretical lattice parameters and volume are $a=4.2699 \AA$, $c=6.4601 \AA, V_{0}=$ $102.0 \AA^{3}$.

\begin{tabular}{lcccr}
\hline \hline Atom & Site & $x$ & $y$ & $z$ \\
\hline $\mathrm{Bi}$ & $1 a$ & 0 & 0 & -0.0258 \\
$\mathrm{Br}$ & $1 b$ & $1 / 3$ & $2 / 3$ & 0.2666 \\
$\mathrm{Te}$ & $1 c$ & $2 / 3$ & $1 / 3$ & 0.7036 \\
\hline \hline
\end{tabular}

compounds under pressure are well described [43]. Theoretical lattice parameters and atomic positions of $\mathrm{BiTeBr}$ at room pressure are summarized in Table I.

Lattice dynamics calculations of the phonon modes were also performed in the $P 3 m 1$ phase at the BZ center $(\Gamma$ point) with the direct force constant approach [44]. Highly converged results on forces are required for the calculation of the dynamical matrix. The construction of the dynamical matrix at the $\Gamma$ point of the BZ involves separate calculations of the forces in which a fixed displacement from the equilibrium configuration of the atoms within the cell is considered. The symmetry of the structure allows reducing the number of such independent displacements and therefore reducing the computational effort in the study of the structures considered in this work. Diagonalization of the dynamical matrix provides both the frequencies of the normal modes and their polarization vectors. It allows identification of the irreducible representation and the character of the phonons modes at the $\Gamma$ point, information that is not readily accessible in the present experiment. In this work, we provide and discuss the calculated frequencies and pressure coefficients of the Raman-active modes for the low-pressure phase of BiTeBr. Moreover, phonon dispersion curves along the whole BZ were calculated, at different pressures, with the supercell method [44].

\section{RESULTS AND DISCUSSION}

\section{A. Characterization of $\mathrm{BiTeBr}$ at room conditions}

XRD measurements at room conditions [Fig. 1(a)] revealed that $\mathrm{BiTeBr}$ crystallized in a hexagonal-type lattice. Le Bail refinement of XRD data assuming the $P 3 m 1$ (or $C_{3 v}$ ) structure yielded the following lattice parameters of $\mathrm{BiTeBr}$ at room pressure: $a=4.26636(10) \AA, c=6.48643(18) \AA, \mathrm{V}=$ 102.247(5) $\AA^{3}$, and $\chi^{2}=1.80$. These values show good agreement with our theoretical lattice parameters (see Table I) and are slightly larger than those of Refs. [20] and [27], but similar to those from Refs. [21,24,25] despite the fact that in all these works BiTeBr was assumed to crystallize in SG $P \overline{3} m 1$ (or $D_{3 d}$ ). It must be stressed that the lattice parameters of both SG $P \overline{3} m 1$ and SG $P 3 m 1$ phases are very similar. In fact, the two phases differ only by an inversion center and cannot be univocally determined through powder XRD measurements because both phases present the same systematic extinctions.

Fortunately, the $\mathrm{SG} P \overline{3} m 1$ and $\mathrm{SG} P 3 m 1$ phases can be clearly distinguished by vibrational spectroscopy. Group theory predicts nine zone-center modes for BiTeBr with SG 
TABLE II. Experimental and theoretical room-temperature Raman-mode frequencies and pressure coefficients in BiTeBr at room pressure, as obtained by fitting the data using $\omega(P)=\omega_{0}+a_{1} P+a_{2} P^{2}$. The last two columns correspond to experimental frequencies and pressure coefficients in BiTeI from Ref. [15].

\begin{tabular}{|c|c|c|c|c|c|c|c|c|}
\hline \multirow[b]{2}{*}{ Mode } & \multicolumn{6}{|c|}{$\mathrm{BiTeBr}$} & \multicolumn{2}{|c|}{ BiTeI } \\
\hline & $\begin{array}{l}\omega_{0}(\text { th. }) \\
\left(\mathrm{cm}^{-1}\right)\end{array}$ & $\begin{array}{c}a_{1} \text { (th.) } \\
\left(\mathrm{cm}^{-1} / \mathrm{GPa}\right)\end{array}$ & $\begin{array}{c}a_{2} \text { (th.) } \\
\left(\mathrm{cm}^{-1} / \mathrm{GPa}^{2}\right)\end{array}$ & $\begin{array}{c}\omega_{0} \text { (expt.) } \\
\left(\mathrm{cm}^{-1}\right)\end{array}$ & $\begin{array}{c}\mathrm{a}_{1} \text { (expt.) } \\
\left(\mathrm{cm}^{-1} / \mathrm{GPa}\right)\end{array}$ & $\begin{array}{c}\mathrm{a}_{2} \text { (expt.) } \\
\left(\mathrm{cm}^{-1} / \mathrm{GPa}^{2}\right)\end{array}$ & $\begin{array}{c}\omega_{0} \text { (expt.) } \\
\left(\mathrm{cm}^{-1}\right)\end{array}$ & $\begin{array}{c}\mathrm{a}_{1}(\text { expt. }) \\
\left(\mathrm{cm}^{-1} / \mathrm{GPa}\right)\end{array}$ \\
\hline \multirow[t]{2}{*}{$\overline{E^{1}(\mathrm{TO})}$} & 71.0 & 3.8 & -0.14 & $64.4^{\mathrm{a}}$ & $4.3^{\mathrm{a}}$ & $-0.18^{\mathrm{a}}$ & 56 & 3.5 \\
\hline & & & & $63.2^{\mathrm{b}}$ & $4.2^{\mathrm{b}}$ & $-0.33^{b}$ & & \\
\hline$E^{1}(\mathrm{LO})$ & 99.5 & 3.3 & -0.13 & & & & & \\
\hline \multirow[t]{2}{*}{$A_{1}{ }^{1}(\mathrm{TO})$} & 104.0 & 2.7 & 0.08 & $100.8^{\mathrm{a}}$ & $4.5^{\mathrm{a}}$ & $-0.15^{\mathrm{a}}$ & 92 & 4.6 \\
\hline & & & & $105.1^{\mathrm{b}}$ & $2.6^{\mathrm{b}}$ & $0.09^{b}$ & & \\
\hline$A_{1}^{1}(\mathrm{TO})$ & 109.7 & 3.6 & -0.16 & $108.3^{\mathrm{a}}$ & $4.1^{\mathrm{a}}$ & $-0.20^{\mathrm{a}}$ & & \\
\hline \multirow[t]{2}{*}{$E^{2}(\mathrm{TO})$} & 109.8 & 3.5 & -0.15 & $108.3^{\mathrm{a}}$ & $4.1^{\mathrm{a}}$ & $-0.20^{\mathrm{a}}$ & 101 & 3.9 \\
\hline & & & & $108.2^{\mathrm{b}}$ & $4.4^{\mathrm{b}}$ & $-0.26^{b}$ & & \\
\hline$E^{2}(\mathrm{LO})$ & 130.8 & -0.08 & 0.09 & $121.7^{\mathrm{a}}$ & $-1.0^{\mathrm{a}}$ & $0.22^{\mathrm{a}}$ & & \\
\hline \multirow[t]{2}{*}{$A_{1}^{2}(\mathrm{TO})$} & 155.7 & 4.1 & -0.09 & $150.3^{\mathrm{a}}$ & $5.0^{\mathrm{a}}$ & $-0.13^{\mathrm{a}}$ & 148 & 4.1 \\
\hline & & & & $153.7^{\mathrm{b}}$ & $4.6^{\mathrm{b}}$ & $-0.15^{b}$ & & \\
\hline$A_{1}^{2}(\mathrm{LO})$ & 156.9 & 4.0 & -0.09 & $154.0^{\mathrm{a}}$ & $4.9^{\mathrm{a}}$ & $-0.13^{\mathrm{a}}$ & & \\
\hline
\end{tabular}

${ }^{a}$ Vertical $(X X$ or parallel) polarization.

${ }^{\mathrm{b}}$ Horizontal ( $X Y$ or perpendicular) polarization.

$P 3 m 1$ with mechanical representation [45]

$$
\Gamma_{9}=3 A_{1}+3 E,
$$

where $E$ modes (doubly degenerate in frequency) correspond to atomic vibrations in the plane of the layers, while $A_{1}$ modes correspond to vibrations along the $c$ axis perpendicular to the layers [28]. One $A_{1}$ mode and one $E$ mode correspond to the three acoustic frequencies, while the rest are optical modes that are both Raman and infrared (IR) active and show transverse optical (TO) and longitudinal optical (LO) splitting. This results in a total of eight possible Raman-active and IRactive frequencies; i.e., four TO frequencies plus their LO counterparts.

On the other hand, group theory predicts nine vibrational modes for $\mathrm{BiTeBr}$ with $\mathrm{SG} P \overline{3} m 1$ with the mechanical representation

$$
\Gamma_{9}=A_{1 g}+2 A_{2 u}+E_{g}+2 E_{u},
$$

where $E$ modes (doubly degenerate) correspond to atomic vibrations in the plane of the layers, while $A$ modes correspond to vibrations along the $c$ axis perpendicular to the layers. Unlike in the SG P3ml phase, Raman and IR modes in the SG $P-3 m 1$ phase are mutually exclusive so there are only two Raman-active frequencies (those for $A_{1 g}$ and $E_{g}$ modes), and only two IR-active frequencies (those for $A_{2 u}$ and $E_{u}$ modes), with the rest being acoustic modes (one $A_{2 u}$ and one $E_{u}$ ).

Figure 1(b) shows the unpolarized RS spectra of BiTeBr at ambient pressure for two different excitation densities (differing in a factor of 2). Our RS spectra clearly show more than two Raman-active modes and therefore we can state that our BiTeBr sample has $P 3 m 1$ structure [see the theoretical frequencies marked at the bottom of Fig. 1(b)]. A good agreement is found between our experimental and theoretical frequencies for the $P 3 m 1$ phase at room pressure which allows for a tentative assignment of mode symmetries to experimental modes (see Table II). Note that under higher excitation the modes around 120 and $140 \mathrm{~cm}^{-1}$ are enhanced and the main modes around 110 and $155 \mathrm{~cm}^{-1}$ exhibit a small shift to lower frequencies due to a slight sample heating. The mode at $140 \mathrm{~cm}^{-1}$ (barely seen in RS at high pressure) is tentatively attributed to a second-order Raman mode and it is not given in Table II. Furthermore, our experimental RS spectrum at low excitation density is in good agreement with that already reported in Ref. [23] for BiTeBr with $P 3 m 1$ structure. On the contrary, our RS spectrum for BiTeBr is different from that published in Ref. [28]; we have no explanation for this disagreement, but the RS spectrum of Ref. [28] could be that of a laser-damaged sample or be the spectrum of a nonpure or oxidized sample. Note that bismuth tellurohalides are polar semiconductors [26] that are very easy to burn during RS measurements for laser powers above $5 \mathrm{~mW}$ and very prone to suffer surface oxidation at ambient conditions.

In summary, XRD and RS measurements have allowed us to determine the structural parameters and vibrational modes of BiTeBr, thus confirming the BiTeI-type (SG P3m1) structure of our samples at room conditions.

\section{B. XRD measurements in compressed BiTeBr}

XRD patterns of $\mathrm{BiTeBr}$ at selected pressures up to $8 \mathrm{GPa}$ are shown in Fig. S1 of the Supplemental Material [46] which also shows a full weighted Le Bail refinement of the XRD pattern obtained at $0.6 \mathrm{GPa}$ using the BiTeI-type structure (the obtained residuals are also plotted). It can be observed that the initial rhombohedral structure is stable up to $7 \mathrm{GPa}$, where a first-order phase transition can be unambiguously identified by the appearance of new Bragg peaks and the disappearance of other features. The phase transition pressure is similar to that reported for BiTeI [14-16,18]. The onset of the phase transition is placed at $7 \mathrm{GPa}$ but the initial and high-pressure phases coexist up to at least $12 \mathrm{GPa}$. The study of the highpressure phase of this compound is out of the scope of this work. However, we can note that the phase transition is fully 

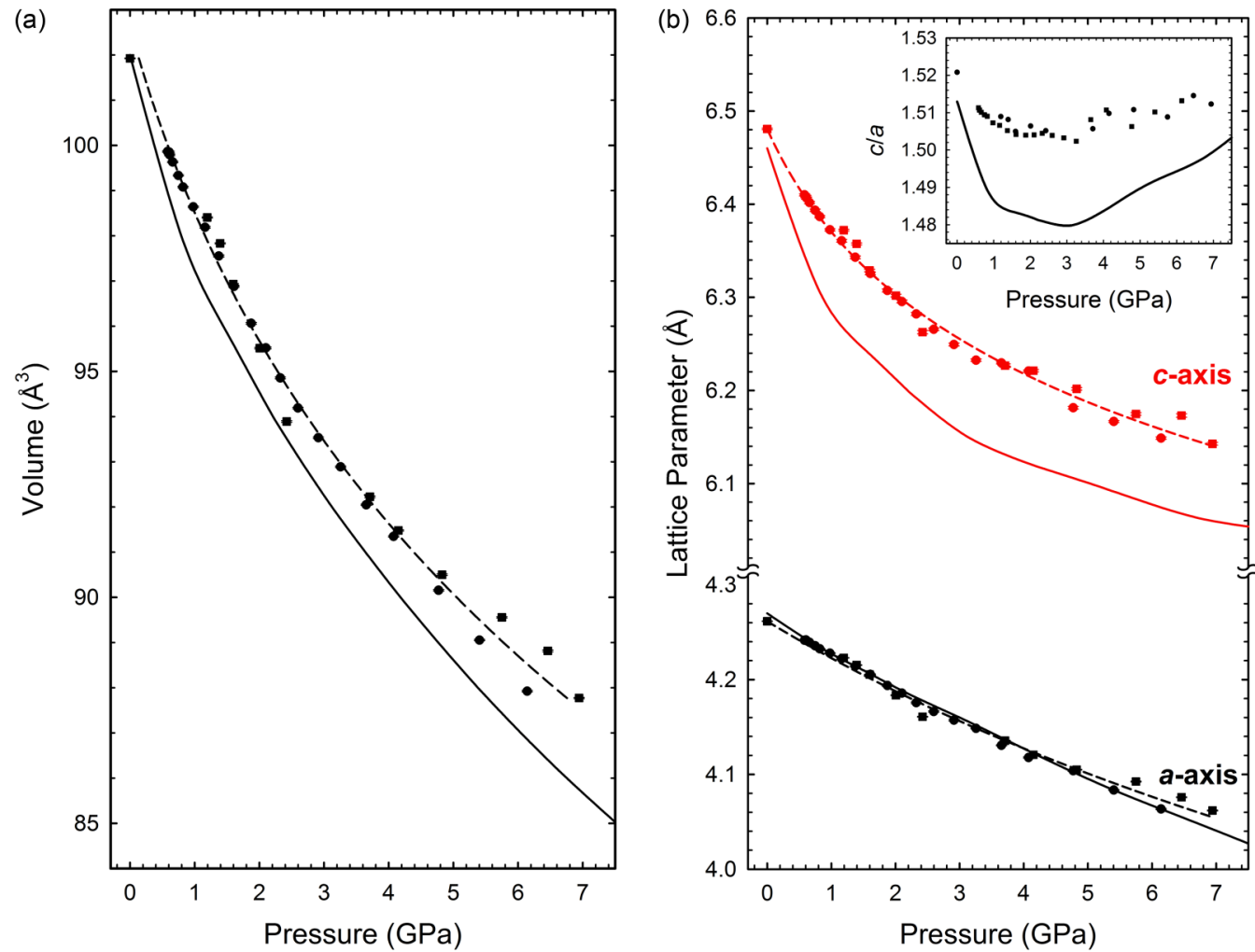

FIG. 2. Pressure dependence of the unit cell volume (a) and lattice parameters (b) of BiTeBr under compression. Circles (squares) represent two different experiments performed using (4:1) methanol/ethanol mixture (silicone oil) as pressure-transmitting medium. Experimental data (symbols) are fitted to a BM-EOS (dashed lines) and compared to theoretical data (solid lines). Inset shows the experimental (symbols) and theoretical (line) $c / a$ ratio.

reversible upon increasing pressure to $20 \mathrm{GPa}$ (see the pattern of the recovered sample at room pressure at the top of Fig. S1).

Figure 2(a) shows the pressure dependence of the unit cell volume in BiTeI-type BiTeBr. As can be observed, the experimental pressure dependence of the volume agrees nicely with that predicted by total-energy ab initio calculations. A fit of experimental data to a third-order Birch-Murnaghan equation of state (BM-EOS) yields an initial volume, bulk modulus, and pressure derivative of $V_{0}=102.6(6) \AA^{3}, B_{0}=20(4) \mathrm{GPa}$, and $B^{\prime}{ }_{0}=11(3)$, respectively. These results are in good agreement with our theoretical results $\left[V_{0}=101.6(3) \AA^{3}, B_{0}=\right.$ 21(1) $\mathrm{GPa}$, and $\left.B_{0}^{\prime}=8.1(7)\right]$. Furthermore, the above values for $\mathrm{BiTeBr}$ are similar to the values of our own experimental and theoretical data for BiTeI [47].

Figure 2(b) displays the pressure dependence of the lattice parameters in BiTeI-type BiTeBr showing a good agreement between experimental and theoretical results. A fit of experimental data to a modified BM-EOS [48] yields $a=$ 4.26636(10) $\mathrm{A}, B_{0 a}=34(1) \mathrm{GPa}$, and $B^{\prime}{ }_{0 a}=2.4(3)$ and $c=$ 6.48643(18), $B_{0 c}=5.9(5) \mathrm{GPa}$, and $B_{0 c}^{\prime}=15.5(5)$. These results yield relative compressibilities of $a$ and $c$ axes of $9.8(3) \times 10^{-3} \mathrm{GPa}^{-1}$ and 57(5) $\times 10^{-3} \mathrm{GPa}^{-1}$. Clearly, the $c$ axis is more compressible than the $a$ axis at room pressure, as expected from the strong initial compression of van der Waals bonds between adjacent layers piled up along the $c$ axis. However, the evolution of the $c / a$ ratio under pressure shown in the inset of Fig. 2(b) shows a change of this trend with pressure with a minimum between 2 and $3 \mathrm{GPa}$ in good agreement with theoretical calculations. We want to stress that the larger compressibility of the $c$ axis than the $a$ axis at room pressure and the existence of a minimum of the $c / a$ ratio in $\mathrm{BiTeBr}$ is similar to previously published results for BiTeI $[14,18]$. In fact, the total experimental $c / a$ change at low pressures is less than 0.1 in $\mathrm{BiTeBr}$ and around 0.2 in BiTeI [14]. This result is in agreement with the larger polarity of $\mathrm{BiTeBr}$ as compared to BiTeI [26], because it can be considered that the stronger the interlayer force the smaller the $c / a$ ratio change.

The large value of the experimental bulk modulus pressure derivative $B^{\prime}{ }_{0}$, which is related to the large value of the experimental $B^{\prime}{ }_{0 c}$, together with the change of the trend of the $c / a$ ratio with increasing pressure, suggests the occurrence of a possible pressure-induced IPT in BiTeBr, as recently observed in some Sb- and Bi-related compounds [49,50]. In order to explore the occurrence of a pressure-induced IPT in $\mathrm{BiTeBr}$, we have analyzed the pressure dependence of the theoretical intralayer $\mathrm{Bi}-\mathrm{Te}$ and $\mathrm{Bi}-\mathrm{Br}$ bond lengths together with the interlayer Te-Br distance (see Fig. S2(a) in [46]). As observed, both $\mathrm{Bi}-\mathrm{Te}$ and $\mathrm{Bi}-\mathrm{Br}$ intralayer distances show a rather monotonic and linear decrease with pressure, whereas the interlayer Br-Te distance shows a strong nonlinear decrease with pressure; i.e., a much larger decrease below $2 \mathrm{GPa}$ than above this pressure. This last behavior can be understood by the strong compression of the space between the layers linked by weak van der Waals forces in comparison to the small compression of the intralayer ionic-covalent bonds below 2 $\mathrm{GPa}$. On the other hand, both interlayer and intralayer bonds compress in a more similar way at higher pressures. The changes in the interlayer and intralayer bonds with increasing 
pressure are related to changes in the atomic positions at 1a (Bi), 1b (Br), and 1c (Te) Wyckoff sites with a free $z$ coordinate (see Fig. S2(b) in [46]). As observed, the theoretical $z$ coordinates of the atomic positions of $\mathrm{Bi}, \mathrm{Br}$, and $\mathrm{Te}$ have a different trend below and above 2-3 GPa. However, all of them evolve in a relatively continuous way and none of them tends either from a high- to a low-symmetry position or from a low- to a high-symmetry position, as in the IPT recently found in $\beta-\mathrm{Bi}_{2} \mathrm{O}_{3}$ [50]. Therefore, our structural data do not provide evidence for the presence of a pressure-induced IPT in $\mathrm{BiTeBr}$ above 2-3 GPa.

\section{Raman scattering of compressed BiTeBr}

Vertically- and horizontally-polarized RS spectra of BiTeBr at different pressures up to 6.1 GPa are shown in Figs. S3(a) and S3(b), respectively, in the Supplemental Information [46]. Different RS spectra were observed at higher pressures, thus confirming the phase transition observed by XRD. Between five and seven Raman-active modes were observed in the low-pressure phase and followed under pressure depending on the polarization. All measured Raman modes shift to higher frequencies with increasing pressure.

The experimental frequencies of the Raman-active modes for both polarizations as a function of pressure are plotted in Figs. 3(a) and 3(b). The experimental values of the frequencies and pressure coefficients are in rather good agreement with our theoretical calculations (see Table II), which show a rather monotonic increase of Raman-active mode frequencies with pressure except for the $E^{2}(\mathrm{LO})$ mode. The frequency of this mode seems to be overestimated in calculations and it slightly decreases below $1 \mathrm{GPa}$ and slightly increases above this pressure. On the other hand, our calculations show an increase of the theoretical LO-TO splitting for the $A_{1}{ }^{1}$ mode below $2 \mathrm{GPa}$ and a subsequent decrease above $2 \mathrm{GPa}$.

It is interesting to compare the Raman mode frequencies and pressure coefficients of $\mathrm{BiTeBr}$ and BiTeI (see Table II).
It can be observed that the measured pressure coefficients for the different modes are rather similar in both compounds. As regards the low-frequency modes, it is known that in layered materials the lowest-frequency $E$ and $A$ modes are usually related to shear vibrations between adjacent layers along the $a-b$ plane and to vibrations of one layer against the others along the $c$ axis, respectively. The low-frequency $E^{1}(\mathrm{TO})$ mode has a similar pressure coefficient as the rest of the optical modes and the low-frequency $A_{1}^{1}$ (TO) mode has not so large a pressure coefficient as expected. A comparison between the behavior of those modes under pressure in these compounds with layered InSe and $\mathrm{GaSe}$ as well as with semiconductors of the $\mathrm{Bi}_{2} \mathrm{Se}_{3}$ family is commented in the Supplemental Information [46]. It can be concluded that the relatively large and similar pressure coefficients of the low-frequency $E^{1}(\mathrm{TO})$ and $A_{1}{ }^{1}(\mathrm{TO})$ modes in $\mathrm{BiTeBr}$ and BiTeI suggest that interlayer forces in these compounds are stronger than common van der Waals forces in other layered compounds. We think that this could likely be due to the strong polarity of bismuth tellurohalides [26]. Moreover, the rather similar pressure coefficients of these two modes in $\mathrm{BiTeBr}$ and BiTeI also suggests that bending and stretching interlayer bonds tend to harden at similar rates with pressure in both compounds; i.e., the anisotropy in the properties along the layers and perpendicular to the layers is not so high as in other layered compounds ( $\mathrm{GaSe}$ and $\mathrm{InSe}$ ) and tends to disappear at a similar rate with increasing pressure in both compounds.

As regards the high-frequency modes, we must note that the high-frequency $E^{2}$ and $A_{1}^{2}$ modes in BiTeBr and BiTeI have high and rather similar pressure coefficients (between 4 and $5 \mathrm{~cm}^{-1} / \mathrm{GPa}$ ), as expected for strong ionic-covalent intralayer bonds. This result is in good agreement with the similar bond distances and bond compressibilities of both intralayer $\mathrm{Bi}-\mathrm{Te}$ and $\mathrm{Bi}-\mathrm{Br}$ distances shown in Fig. S2(a) in [46]. Furthermore, the pressure coefficients of the highfrequency $E$ and $A$ modes in $\mathrm{BiTeBr}$ and BiTeI are larger than those in $\alpha-\mathrm{Sb}_{2} \mathrm{Te}_{3}, \alpha-\mathrm{Bi}_{2} \mathrm{Te}_{3}$, and $\alpha-\mathrm{Bi}_{2} \mathrm{Se}_{3}$ [51-53]. It is noteworthy that a comparison of Grüneisen parameters of
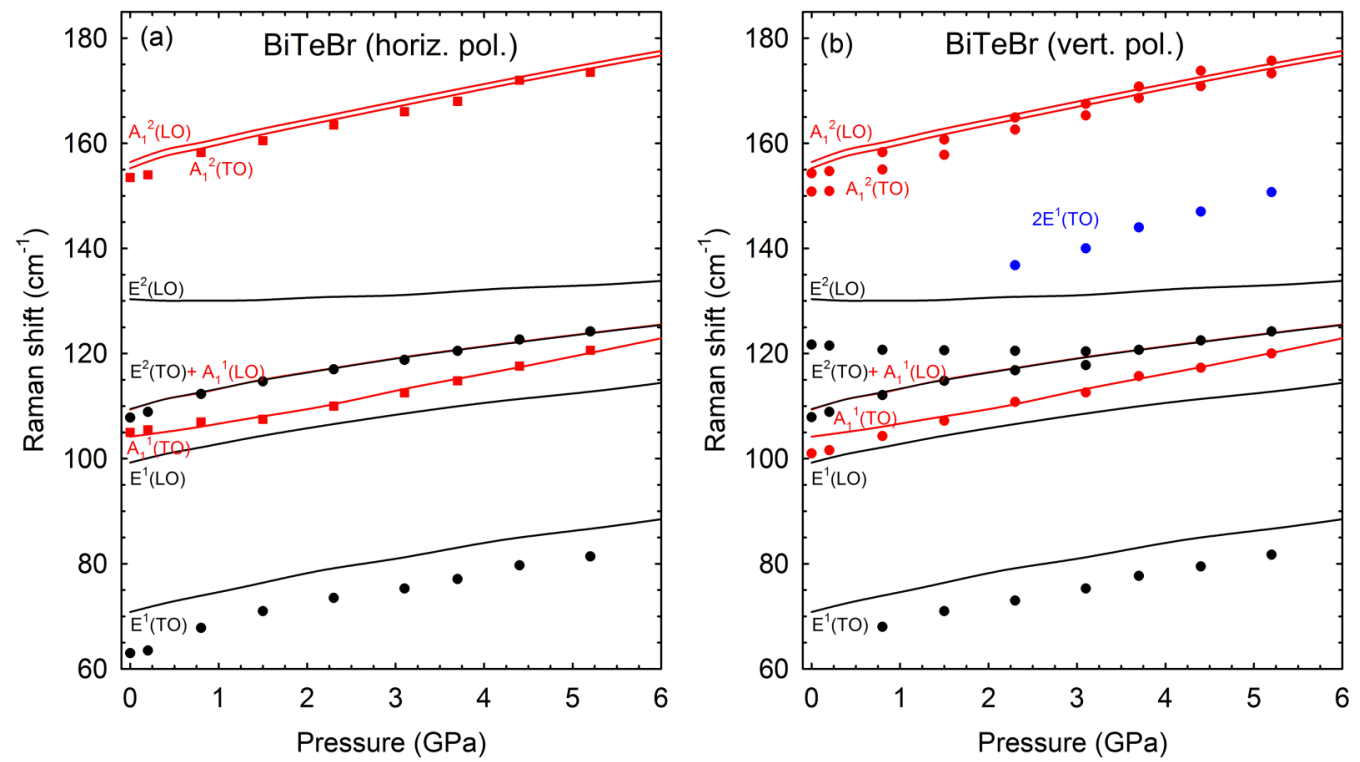

FIG. 3. Pressure dependence of the experimental (symbols) and theoretical (lines) Raman-active mode frequencies in BiTeBr for vertical polarization (a) and horizontal polarization (b). 
the high-frequency Raman modes of both families, assuming bulk moduli around $20 \mathrm{GPa}$ in $\mathrm{BiTe} X$ compounds and around $50 \mathrm{GPa}$ in the $\mathrm{Bi}_{2} \mathrm{Se}_{3}$ family, yields values of around 0.7 for the former and larger than 1.1 for the latter. This result means that the anharmonicity of intralayer ionic-covalent forces in the two families is different, and the intralayer forces evolve in a different way with compression in the two families as already observed for the interlayer forces.

In summary, the comparison of RS measurements under pressure in layered bismuth tellurohalides with other layered compounds indicates that bismuth tellurohalides behave more as three-dimensional materials than other layered semiconductors, like those of the $\mathrm{Bi}_{2} \mathrm{Se}_{3}$ or InSe families. This is in good agreement with the reported difficulty of exfoliating layers in bismuth tellurohalides [26,54]. This behavior can be understood if we consider that layers in bismuth tellurohalides are asymmetric (Te-Bi-Br) while in the other two families they are symmetric (Se-Bi-Se-Bi-Se or Se-In-In-Se). The asymmetric layer in bismuth tellurohalides results in asymmetric charges in the layer [26,54]. The different electric charge in the Te plane than in the $\mathrm{Br}$ plane could contribute to a partially ionic character of the interlayer forces, which is not present in the other two families of layered materials, so that the interlayer forces are stronger in the bismuth tellurohalides than in other layered semiconductor families with symmetrical layers. This leads to a smaller anisotropy in the bismuth tellurohalides than in other layered semiconductor families with symmetrical layers.

As regards the discussion of a possible IPT in BiTeBr, the small changes in frequencies and linewidths around 1-2 GPa are the only clues in our RS measurements which could suggest a possible pressure-induced IPT. This situation contrasts with recent results in $\beta-\mathrm{Bi}_{2} \mathrm{O}_{3}$ where a zone-center

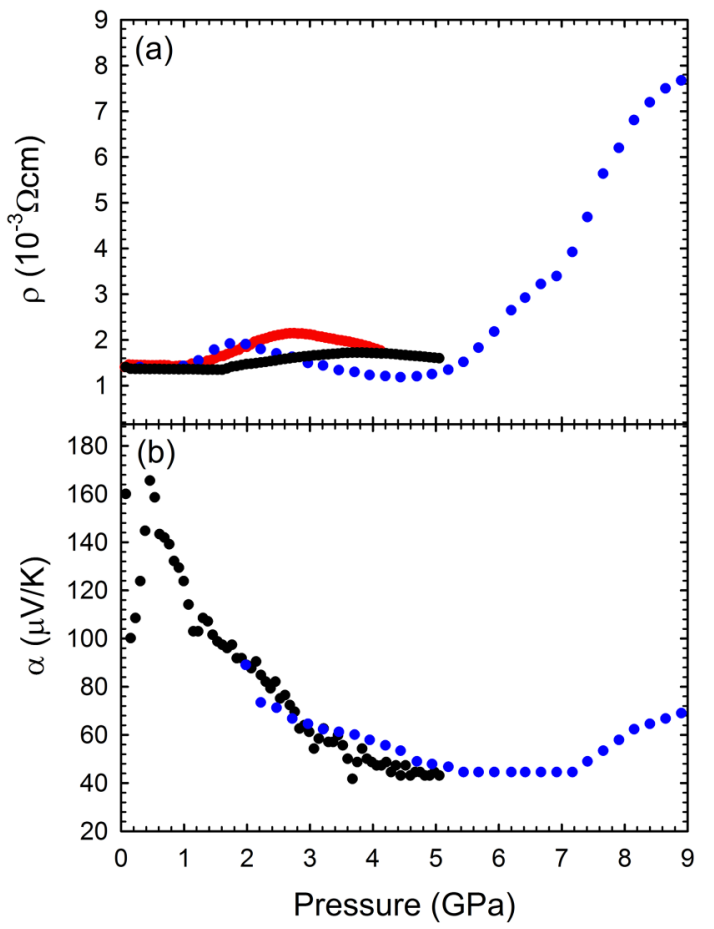

soft phonon mode decreasing to zero frequency at the IPT was recently reported [50]. In order to find whether there is a soft mode which could be indicative of a second-order IPT, we have performed lattice dynamics calculations along the whole BZ at different pressures. Figures S5(a) and S5(b) in the Supplemental Material [46] show the phonon dispersion curves of $\mathrm{BiTeBr}$ at room pressure and $2.2 \mathrm{GPa}$. It can be clearly observed that there is no soft mode that triggers a second-order IPT along the whole BZ. Therefore, we conclude that a second-order pressure-induced IPT is not present in hydrostatically compressed $\mathrm{BiTeBr}$ and the same situation is thought to occur in hydrostatically compressed BiTeI according to recent $a b$ initio calculations [47].

Finally, we must mention that in a previous work [15] it was reported that the $E^{2}(\mathrm{TO})$ mode, usually the most intense one in BiTeBr and BiTeI [52], showed a strong decrease in linewidth between room pressure and $4 \mathrm{GPa}$. This feature was interpreted as indicative of the occurrence of an ETT near $4 \mathrm{GPa}$, similar to that observed in several topological insulators $\left(\mathrm{Bi}_{2} \mathrm{Se}_{3}, \mathrm{Bi}_{2} \mathrm{Te}_{3}\right.$, and $\mathrm{Sb}_{2} \mathrm{Te}_{3}$ ) [51-53]. Our measurements of the full width at half maximum of this Raman mode in BiTeBr (see Fig. S4 in [46]) also shows the same behavior but it was interpreted as due to its particular morphology of the two-phonon density of states and the way the frequency of the first-order mode sweeps the two-phonon density of states as pressure increases [54].

\section{Transport measurements under pressure}

In order to confirm whether a possible pressure-induced ETT exists in our BiTeI-type BiTeBr samples we have performed transport measurements under pressure. Figure 4(a) shows the pressure dependence of the electric resistivity of

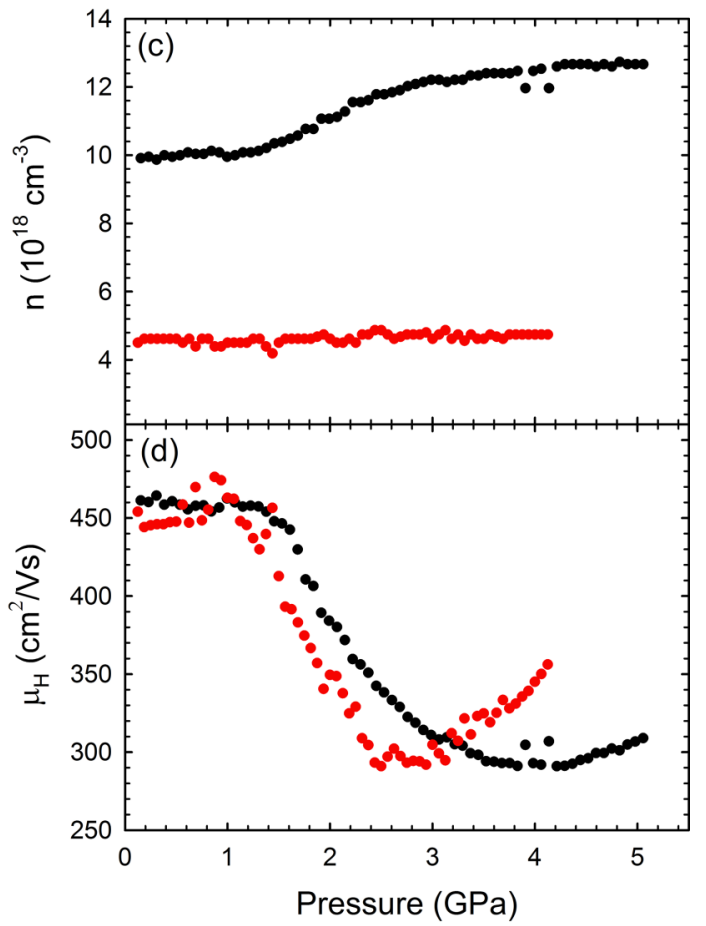

FIG. 4. Pressure dependence of the resistivity (a) thermoelectric power (b), electron concentration (c), and electron mobility (d) in BiTeBr at room temperature. Black (red) symbols correspond to samples with high (low) electron concentration. Blue symbols refer to measurements in the sample with high electron concentration performed with smaller Tungsten Carbide anvils. 
$\mathrm{BiTeBr}$ at room temperature for three samples with different carrier concentration. Resistivity increases by about $50 \%$ up to $1.7 \mathrm{GPa}$ and then decreases by the same amount between 1.7 and $5 \mathrm{GPa}$. Above this pressure, a quicker increase in resistivity is found, which can be attributed to the creation of defects, precursors of the phase transition above 6-7 GPa as observed in XRD and RS measurements. The pressure increase of the resistivity in $\mathrm{BiTeBr}$ at low pressure contrasts with the decrease reported for the resistivity of relatively highly doped $\mathrm{BiTeI}$ samples at room temperature up to $3 \mathrm{GPa}[55,56]$. Before explaining the behavior of resistivity with increasing pressure in $\mathrm{BiTeBr}$ we will analyze first the pressure dependence of the Seebeck coefficient and of the electron concentration and mobility in our samples.

Figure 4(b) shows the pressure dependence of the Seebeck coefficient obtained from thermopower measurements. The Seebeck coefficient exhibits a monotonic decrease up to 4-5 GPa and remains constant above this pressure up to $7 \mathrm{GPa}$, where the first-order phase transition takes place as shown in XRD and RS measurements. This last result is in good agreement with the increase of resistivity above 6-7 GPa. Figure 4(c) shows the pressure dependence of the electron concentration for two BiTeBr samples, as extracted from the Hall coefficient. The electron concentration is approximately constant in the pressure range studied for the sample with lower electron concentration (less degenerate). However, the sample with higher electron concentration (more degenerate) exhibits a monotonic increase of the electron concentration by approximately $30 \%$ between 1 and $4 \mathrm{GPa}$. Figure 4(d) shows the pressure dependence of the electron Hall mobility for the same two samples. As observed, the electron mobility remains almost constant between room pressure and 1-1.5 GPa, then decreases, and after a minimum between 2.5 and $4 \mathrm{GPa}$ increases again.

For the analysis of the results of transport measurements in bismuth tellurohalides is necessary to know the pressure dependence of the electronic band structure. Thus, we have calculated the pressure dependence of the electronic band structure of BiTeBr and BiTeI with SG P $3 m 1$. In this respect, we must note that the evolution of the electronic band structure of $\mathrm{BiTeBr}$ with increasing pressure shows similar trends to those already reported for BiTeI $[13,18]$. At room pressure, BiTeBr is a quasidirect band gap semiconductor whose calculated band gap near the $A$ point, underestimated by DFT calculations, is around $0.27 \mathrm{eV}$ [see Fig. S6(a) in the Supplemental Material [46]] . This value can be compared to its experimental band gap between 0.55 and $0.59 \mathrm{eV}[27,57,58]$. Similarly, BiTeI has a theoretical quasidirect band gap of $0.15 \mathrm{eV}$ at room pressure which compares to an experimental band gap of $0.39 \mathrm{eV}$ [59]. The quasidirect band gap of BiTeBr exhibits a strong negative pressure coefficient (around $-0.09 \mathrm{eV} / \mathrm{GPa}$ ) near room pressure, which is slightly smaller than that of BiTeI $(-0.12 \mathrm{eV} / \mathrm{GPa})[18,46]$. However, the band gap pressure coefficient decreases when the band gap is almost closed; i.e., prior to the TQPT. Once the band gap is closed, an increase of the band gap energy is theoretically predicted in our calculations, in good agreement with previous calculations [18].

The band gap energy difference between BiTeBr and BiTeI and their rather similar pressure coefficients are important in order to interpret transport measurements in both compounds. This makes a difference in relation to the discussed observation of the pressure-induced TQPT in BiTeI $[13,14,16,18]$. In this respect, the closure of the band gap is likely to occur in BiTeI above 3.0 GPa, while in BiTeBr it should not occur below $6 \mathrm{GPa}$; i.e., very close to the pressure for the transition to the high-pressure phase. We note that at $2 \mathrm{GPa}$, the quasidirect band gap of $\mathrm{BiTeBr}$ should be around $0.39 \mathrm{eV}$; i.e., it is almost as large as the band gap energy of BiTeI at ambient pressure. Therefore, changes observed in resistivity above $2 \mathrm{GPa}$ in BiTeBr can hardly be associated to the TQPT related to the closure of the band gap.

Band structure calculations of $\mathrm{BiTeBr}$ show considerable changes in the dispersion in $k$ space of the valence band maxima (VBMs) and conduction band minima (CBMs) near the $A$ point of the BZ upon decreasing the band gap energy with increasing pressure. At room pressure, bands near the band gap energy show parabolic dispersion in $k$ space [see Fig. S6(a)]; however, with increasing pressure and band gap closening the bands tend to show linear dispersion as in a Dirac cone [see Fig. S6(b)]. A similar pressure dependence of the $k$ dispersion of the bands forming the quasidirect band gap has been found in our band structure calculations for BiTeI [47]. This change of the band dispersion is consistent with recent estimations obtained from Shubnikov-de Haas oscillations in BiTeI under pressure, where samples with a high electron concentration have been found to cross the inner Dirac cone of the conduction band at the $A$ point at high pressures [56].

In order to understand the different behavior of transport properties in the two compounds it is also necessary to estimate the Fermi level and its pressure dependence in BiTeBr. According to Ref. [59], the CBM has a toroidal constant energy surface around the three momentum directions. The density of states above the toroidal CBM is constant and is given by the following equation:

$$
g(E)=\frac{\left(m^{*}\right)^{3 / 2} \sqrt{E_{0}}}{\sqrt{2} \pi \hbar^{3}}=g_{0},
$$

where $E_{0}$ is the Rashba energy and $m^{*}$ is the average effective mass in a plane containing the $c$ axis. The constant density of states can also be written as a function of the radius $\left(k_{0}\right)$ of the toroid in the $k$ space [60]:

$$
g_{0}=\frac{m^{*} k_{0}}{\pi \hbar^{2}}
$$

with a radius of $0.043 \AA^{-1}[4,59]$ and an effective mass $0.2 m_{0}$ [27], so that the density of states is about $3.6 \times 10^{20} \mathrm{~cm}^{-3} \mathrm{eV}^{-1}$ at room pressure. For a constant density of states $g_{0}$, the Fermi level energy as a function of the electron concentration $(n)$, with respect to the $\mathrm{CBM}$, is given by a simple analytical expression [60]:

$$
E_{F}-E_{C B M}=k_{B} T \ln \left(e^{n / g_{0} k_{B} T}-1\right) .
$$

According to this expression, the Fermi level reaches the CBM for an electron concentration of $6.3 \times 10^{18} \mathrm{~cm}^{-3}$. Therefore, the Fermi level in our samples is $10 \mathrm{meV}$ below and $17 \mathrm{meV}$ above the CBM for samples with electron concentrations of $4.7 \times 10^{18} \mathrm{~cm}^{-3}$ and $10^{19} \mathrm{~cm}^{-3}$, respectively. Consequently, the Fermi level at room pressure is well below the energy of the Dirac point (Rashba energy) at the $A$ point. 
Note that a difference in energy of the order of $100 \mathrm{meV}$ is estimated between the CBM and the Dirac point at the A point of the BZ according to our band structure calculations.

Since the electron concentration does not change under pressure for one sample and increases by $30 \%$ in the other, the pressure evolution of the Fermi level is determined by the density of states that, in turn, depends on the effective mass. According to the $\mathrm{k} \cdot \mathrm{p}$ model, the effective mass is proportional to the band gap, so the negative band gap pressure coefficient $(-0.09 \mathrm{eV} / \mathrm{GPa})$ in $\mathrm{BiTeBr}$ should lead to a decrease of the effective mass with pressure and consequently to a decrease of the density of states. Therefore, as the density of states decreases at constant electron concentration, the Fermi level moves to higher energies with increasing pressure. Note that the Fermi level crosses the CBM when the exponential in Eq. (5) becomes larger than 2. The decrease of the band gap energy in BiTeBr by a factor of 2 between room pressure and 3 GPa leads to a similar factor in the decrease of the effective mass. Thus, from Eq. (5) it follows that the Fermi level at 3 GPa would be 15 and $71 \mathrm{meV}$ above the CBM for the samples with low and high electron concentration, respectively. This is in good agreement with the observed slight decrease of the Seebeck coefficient under pressure [Fig. 4(c)], which also suggests that the material becomes more degenerate under pressure. In any case, despite the fact that our BiTeBr samples become more degenerate at high pressures, the Fermi level is well below the Dirac point and we can thus exclude that the resistivity behavior above $2-3 \mathrm{GPa}$ is related to an ETT due to the crossing of the Fermi level above the inner Dirac cone of the conduction band at the $A$ point. We should also stress that this conclusion is based on the assumption of parabolic dispersion above the CBM. When the band dispersion becomes linear as the band gap decreases, the density of states is no longer constant above the CBM and increases linearly with energy. This change leads to a smaller rate of increase of the Fermi level above the CBM, thus supporting our conclusion that the Fermi level does not cross the Dirac point with increasing pressure in BiTeBr.

With all these considerations in mind, and taking into account that the electron mobility $\mu$, mainly determined by electrons at the Fermi level, is proportional to the relaxation time $\tau$ and inversely proportional to the effective mass $m^{*}$, the pressure dependence of $\mu$ can be understood as follows. Regarding $\tau$, we can reasonably assume that $\tau$ is controlled by phonon scattering, which is more effective for electrons of larger electron kinetic energy. The transition from nondegenerate to degenerate behavior in $\mathrm{BiTeBr}$, due to the upward shift of the Fermi level as pressure increases, leads to a larger weight of high-energy electrons as pressure increases; therefore, the larger phonon scattering of high-energy electrons results in a smaller $\tau$ and smaller $\mu$ as pressure increases.

Concerning $m^{*}$, its value would increase or decrease with pressure depending on the balance of two pressure effects on the CBM: (i) the $\mathrm{k} \cdot \mathrm{p}$ model leads to a proportional decrease of $m^{*}$ as the band gap decreases; and (ii) the band nonparabolicity increases as the band gap decreases; i.e., the $E(k)$ relation becomes more linear, which increases $m^{*}$ for states above the CBM. At room pressure, our BiTeBr sample is either nondegenerate or weakly degenerate and $m^{*}$ is determined by an average of electrons, which include electrons at the CBM with relatively low effective mass. As pressure increases, the electron gas becomes more degenerate and the Fermi level goes above the CBM so $m^{*}$ is more and more determined by electrons with larger effective mass than those at the CBM. This means that at low pressures the decrease of $m^{*}$ due to the decrease of the band gap is partially counterbalanced by the increase of $m^{*}$ due to the increase of the Fermi level, resulting in a constant $\mu$. On the other hand, as pressure increases the increase of the band nonparabolicity should give an increase of $m^{*}$ and a decrease of $\mu$ in the medium-pressure range. Finally, the overall decrease of $m^{*}$ at high pressures due to band gap decrease as the material approaches the band gap closure compensates the effect of nonparabolicity and the increase of $\tau$, thus leading to a final increase of $\mu$ at high pressures.

Finally, we could attribute the increase (decrease) of the resistivity in BiTeBr below (above) 2 GPa to the initially constant electron concentration and mobility with pressure and the decrease (increase) of the electron mobility below (above) $2 \mathrm{GPa}$. In this way, we can conclude that the pressure dependence of the electrical properties of $\mathrm{BiTeBr}$ and BiTeI under compression should behave quite similarly at low pressures. Note that an increase of the Fermi level with increasing pressure is observed in both samples, and a possible crossing of the Fermi level with the inner Dirac cone of the conduction band at the $A$ point could occur in both semiconductors depending on the initial position of the Fermi level, which depends on the initial electron concentration. However, the two semiconductors have very different band gaps, which could very much influence the electric properties with increasing pressure, since the closing of the band gap in BiTeI above 3.5 GPa does not occur in BiTeBr.

\section{CONCLUSIONS}

We have experimentally and theoretically characterized the structural and vibrational properties of $\mathrm{BiTeBr}$ samples at ambient conditions and found that $\mathrm{BiTeBr}$ crystallized in the SG $P 3 m 1$ structure. The experimental structural, vibrational, and electrical properties of compressed $\mathrm{BiTeBr}$ have been interpreted thanks to $a b$ initio calculations. As a layered semiconductor, $\mathrm{BiTeBr}$ exhibits anisotropic compression and undergoes changes in different properties near $2 \mathrm{GPa}$. This anisotropy is smaller than in BiTeI, likely due to the larger charge polarity of interlayer bonds in BiTeBr. Moreover, we have demonstrated that the anisotropy of bismuth tellurohalides is much smaller than in other layered semiconductors. Our study shows that the changes observed can be ascribed to the different interlayer compressibility as pressure increases. Furthermore, the changes in the electrical properties of $\mathrm{BiTeBr}$ have been explained by the complex pressure dependence of the electron effective mass in this compound, which must also apply to BiTeI. We hope the present work will further stimulate new works in bismuth tellurohalides under high pressure in order to understand their exotic properties.

\section{ACKNOWLEDGMENTS}

This work has been performed under financial support from Spanish MINECO under Projects No. MAT2013-46649-C4- 
2/3-P and MAT2015-71070-REDC. This publication is the outcome of "Programa de Valoración y Recursos Conjuntos de $\mathrm{I}+\mathrm{D}+\mathrm{i}$ VLC/CAMPUS" and has been financed by the Spanish Ministerio de Educación, Cultura y Deporte as part of "Programa Campus de Excelencia Internacional" through Projects
No. SP20140701 and No. SP20140871. Supercomputer time has been provided by the Red Española de Supercomputación (RES) and the MALTA cluster. J.A.S. acknowledges the "Juan de la Cierva" fellowship program for financial support.
[1] K. Ishizaka, M. S. Bahramy, H. Murakawa, M. Sakano, T. Shimojima, T. Sonobe, K. Koizumi, S. Shin, H. Miyahara, A. Kimura, K. Miyamoto, T. Okuda, H. Namatame, M. Taniguchi, R. Arita, N. Nagaosa, K. Kobayashi, Y. Murakami, R. Kumai, Y. Kaneko, Y. Onose, and Y. Tokura, Nat. Mater. 10, 521 (2011).

[2] A. Crepaldi, L. Moreschini, G. Autès, C. Tournier-Colletta, S. Moser, N. Virk, H. Berger, P. Bugnon, Y. J. Chang, K. Kern, A. Bostwick, E. Rotenberg, O. V. Yazyev, and M. Grioni, Phys. Rev. Lett. 109, 096803 (2012).

[3] G. Landolt, S. V. Eremeev, Y. M. Koroteev, B. Slomski, S. Muff, T. Neupert, M. Kobayashi, V. N. Strocov, T. Schmitt, Z. S. Aliev, M. B. Babanly, I. R. Amiraslanov, E. V. Chulkov, J. Osterwalder, and J. H. Dil, Phys. Rev. Lett. 109, 116403 (2012).

[4] M. Sakano, M. S. Bahramy, A. Katayama, T. Shimojima, H. Murakawa, Y. Kaneko, W. Malaeb, S. Shin, K. Ono, H. Kumigashira, R. Arita, N. Nagaosa, H. Y. Hwang, Y. Tokura, and K. Ishizaka, Phys. Rev. Lett. 110, 107204 (2013).

[5] Y. L. Chen, M. Kanou, Z. K. Liu, H. J. Zhang, J. A. Sobota, D. Leuenberger, S. K. Mo, B. Zhou, S.-L. Yang, P. S. Kirchmann, D. H. Lu, R. G. Moore, Z. Hussain, Z. X. Shen, X. L. Qi, and T. Sasagawa, Nat. Phys. 9, 704 (2013).

[6] F. X. Xiang, X. L. Wang, M. Veldhorst, S. X. Dou, and M. S. Fuhrer, Phys. Rev. B 92, 035123 (2015).

[7] M. S. Bahramy, R. Arita, and N. Nagaosa, Phys. Rev. B 84, 041202(R) (2011).

[8] S. V. Eremeev, I. A. Nechaev, and E. V. Chulkov, JETP Lett. 96, 437 (2012).

[9] Z.-Y. Zhu, Y.-C. Cheng, and U. Schwingenschlögl, New J. Phys. 15, 023010 (2013).

[10] E .I Rashba, Sov. Phys.-Solid State 2, 1109 (1960).

[11] C. Nayak, S. H. Simon, A. Stern, M. Freedman, and S. Das Sarma, Rev. Mod. Phys. 80, 1083 (2008).

[12] J. Alicea, Y. Oreg, G. Refael, F. v. Oppen, and M. P. A. Fisher, Nat. Phys. 7, 412 (2011).

[13] M. S. Bahramy, B.-J. Yang, R. Arita, and N. Nagaosa, Nat. Commun. 3, 679 (2012).

[14] X. X. Xi, C. L. Ma, Z. X. Liu, Z. Q. Chen, W. Ku, H. Berger, C. Martin, D. B. Tanner, and G. L. Carr, Phys. Rev. Lett. 111, 155701 (2013).

[15] Yu. S. Ponosov, T. V. Kuznetsova, O. E. Tereshchenko, K. A. Kokh, and E. V. Chulvkov, JETP Lett. 98, 557 (2013).

[16] M. K. Tran, J. Levallois, P. Lerch, J. Teyssier, A. B. Kuzmenko, G. Autès, O. V. Yazyev, A. Ubaldini, E. Giannini, D. van der Marel, and A. Akrap, Phys. Rev. Lett. 112, 047402 (2014).

[17] I. P. Rusinov, I. A. Nechaev, S. V. Eremeev, C. Friedrich, S. Blügel, and E. V. Chulkov, Phys. Rev. B 87, 205103 (2013).

[18] Y. Z. Chen, X. X. Xi, W. L. Yim, F. Peng, Y. C. Wang, H. Wang, Y. M. Ma, G. T. Liu, C. L. Sun, C. L. Ma, Z. Q. Chen, and H. Berger, J. Phys. Chem. C 117, 25677 (2013).
[19] J. J. Ying, V. V. Struzhkin, A. F. Goncharov, H.-K. Mao, F. Chen, X.-H. Chen, A. G. Gavriliuk, and X.-J. Chen, arXiv:1501.06203.

[20] E. Dönges, Z. Anorg. Allg. Chem. 265, 56 (1951).

[21] A. V. Shevelkov, E. V. Dikarev, R. V. Shpanchenko and B. A. Popovkin, J. Solid State Chem. 114, 379 (1995).

[22] S. V. Eremeev, I. P. Rusinov, I. A. Nechaev, and E. V. Chulkov, New J. Phys. 15, 075015 (2013).

[23] A. Akrap, J. Teyssier, A. Magrez, P. Bugnon, H. Berger, A. B. Kuzmenko, and D. van der Marel, Phys. Rev. B 90, 035201 (2014).

[24] V. A. Kubalchinskii, V. G. Kytin, Z. V. Lavrukhina, A. N. Kutnesov, and A. V. Shevelkov, Semiconductors 44, 1548 (2010).

[25] V. A. Kubalchinskii, V. G. Kytin, A. A. Kudryashov, A. N. Kutnesov, and A. V. Shevelkov, J. Solid State Chem. 193, 154 (2012).

[26] Y. D. Ma, Y. Dai, W. Wei, X. R. Li, and B. B. Huang, Phys. Chem. Chem. Phys. 16, 17603 (2014).

[27] M. Matyáš, J. Horák and B. Klubíčková, Phys. Status Solidi A 61, 419 (1980).

[28] F. Fauth, I. Peral, C. Popescu, and M. Knapp, Powder. Diffr. 28, S360 (2013).

[29] A. P. Hammersley, S. O. Svensson, M. Hanfland, A. N. Fitch, and D. Hausermann, High Press. Res. 14, 235 (1996).

[30] A. C. Larson and R. B. von Dreele, Los Alamos National Laboratory Report LAUR 86, p. 748 (2004) (unpublished).

[31] B. H. Toby, J. Appl. Crystallogr. 34, 210 (2001).

[32] K. Momma and F. Izumi, J. Appl. Crystallogr. 44, 1272 (2011).

[33] A. Dewaele, P. Loubeyre, and M. Mezouar, Phys. Rev. B 70, 094112 (2004).

[34] G. J. Piermarini, S. Block, and J. D. Barnett, J. Appl. Phys. 44, 5377 (1973).

[35] D. Errandonea, Y. Meng, M. Somayazulu, and D. Hausermann, Physica B 355, 116 (2005).

[36] K. Syassen, High Press. Res. 28, 75 (2008).

[37] D. Errandonea, A. Segura, D. Martínez-García, and V. MuñozSan José, Phys. Rev. B 79, 125203 (2009).

[38] D. Errandonea, D. Martínez-García, A. Segura, J. Ruiz-Fuertes, R. Lacomba-Perales, V. Fages, A. Chevy, L. Roa, and V. MuñozSan José, High Press. Res. 26, 513 (2006).

[39] P. Hohenberg and W. Kohn, Phys. Rev. 136, B864 (1964).

[40] G. Kresse and J. Hafner, Phys. Rev. B 47, 558 (1993); 49, 14251 (1994); G. Kresse and J. Furthmüller, Comput. Mater. Sci. 6, 15 (1996); Phys. Rev. B 54, 11169 (1996).

[41] P. E. Blöchl, Phys. Rev. B 50, 17953 (1994); G. Kresse and D. Joubert, 59, 1758 (1999).

[42] J. P. Perdew, A. Ruzsinszky, G. I. Csonka, O. A. Vydrov, G. E. Scuseria, L. A. Constantin, X Zhou, and K. Burke, Phys. Rev. Lett. 100, 136406 (2008). 
[43] A. Mujica, A. Rubio, A. Muñoz, and R. J. Needs, Rev. Mod. Phys. 75, 863 (2003).

[44] K. Parlinski, computer code PHONON., http://wolf.ifj.edu.pl/ phonon.

[45] E. Kroumova, M. I. Arroyo, J. M. Perez-Mato, A. Kirov, C. Capillas, S. Ivantchev, and H. Wondratschek, Phase Transitions 76, 155 (2003).

[46] See Supplemental Material at http://link.aps.org/supplemental/ 10.1103/PhysRevB.93.024110 for details of comments about the comparison between the behavior of low-frequency $E^{1}$ (TO) and $A_{1}{ }^{1}(\mathrm{TO})$ modes under pressure in $\mathrm{BiTeBr}$ and $\mathrm{BiTeI}$ with that in layered $\mathrm{InSe}$ and $\mathrm{GaSe}$ and semiconductors of the $\mathrm{Bi}_{2} \mathrm{Se}_{3}$ family, and for additional figures.

[47] A. Muñoz (private communication).

[48] R. J. Angel, High-Temperature and High-Pressure Crystal Chemistry, Reviews in Mineralogy and Geochemistry Vol. 41 (2000), p. 35.

[49] A. L. J. Pereira, L. Gracia, D. Santamaría-Pérez, R. Vilaplana, F. J. Manjón, D. Errandonea, M. Nalin, and A. Beltrán, Phys. Rev. B 85, 174108 (2012).

[50] A. L. J. Pereira, J. A. Sans, R. Vilaplana, O. Gomis, F. J. Manjón, P. Rodríguez-Hernández, A. Muñoz, C. Popescu, and A. Beltrán, J. Phys. Chem. C 118, 23189 (2014).

[51] R. Vilaplana, O. Gomis, F. J. Manjón, A. Segura, E. PérezGonzález, P. Rodríguez-Hernández, A. Muñoz, J. González, V. Marín-Borrás, V. Muñoz-Sanjosé, C. Drasar, and V. Kucek, Phys. Rev. B 84, 104112 (2011).
[52] O. Gomis, R. Vilaplana, F. J. Manjón, P. Rodríguez-Hernández, E. Pérez-González, A. Muñoz, V. Kucek, and C. Drasar, Phys. Rev. B 84, 174305 (2011).

[53] R. Vilaplana, D. Santamaría-Pérez, O. Gomis, F. J. Manjón, J. González, A. Segura, A. Muñoz, P. Rodríguez-Hernández, E. Pérez-González, V. Marín-Borrás, V. Muñoz-Sanjose, C. Drasar, and V. Kucek, Phys. Rev. B 84, 184110 (2011).

[54] L. Moreschini, G. Autès, A. Crepaldi, S. Mosera, J. C. Johannsen, K. S. Kima, H. Berger, Ph. Bugnon, A. Magrez, J. Denlinger, E. Rotenberg, A. Bostwick, O. V. Yazyev, and M. Grionic, J. Electron Spectrosc. Relat. Phenom. 201, 115 (2015).

[55] D. VanGennep, S. Maiti, D. Graf, S. W. Tozer, C. Martin, H. Berger, D. L. Maslov, and J. J. Hamlin, J. Phys.: Condens. Matter 26, 342202 (2014).

[56] T. Ideue, J. G. Checkelsky, M. S. Bahramy, H. Murakawa, Y. Kaneko, N. Nagaosa, and Y. Tokura, Phys. Rev. B 90, 161107 (2014).

[57] J. Horák and H. Rodot, C. R. Seances Acad. Sci., Ser. B 267, 1427 (1968).

[58] L. V. Onopko, V. V. Onopko, D. V. Chepur, N. I. Dovgoshei, I. D. Turyanitsa, and N. F. Zayachkovskaya, Izv. Ross. Akad. Nauk 10, 1137 (1974).

[59] L. H. Wu, J. Yang, S. Y. Wang, P. Wei, J. H. Yang, W. Q. Zhang, and L. D. Chen, Phys. Rev. B 90, 195210 (2014).

[60] D. Errandonea, A. Segura, F. J. Manjón, A. Chevy, E. Machado, G. Tobías, P. Ordejón, and E. Canadell, Phys. Rev. B 71, 125206 (2005). 